Latino older adults were similar to Whites in odds of cognitive decline (OR Latino 1.3; 95\% CI 0.9-1.7). Conclusions: African American adults aged 65 and above were more likely to experience cognitive decline compared to White older adults after accounting for demographics, socioeconomics, comorbidity, and their baseline cognitive function. There was no difference between Latino older adults and Whites in rates of cognitive decline. Future research to identify ways to reduce cognitive decline, particularly for racially-diverse groups, are needed.

\section{O4-05-06 PREVALENCE RATES FOR DEMENITA AND ALZHEIMER'S DISEASE IN ELDERLY AFRICAN AMERICANS IN INDIANAPOLIS: 1992 VS 2001}

Kathleen S. Hall ${ }^{1,2}$, Jianzhao Shen ${ }^{1}$, Sujuan Gao $^{1}$, Jill R. Murrell ${ }^{1}$, Valerie Smith-Gamble ${ }^{1}$, Adesola Ogunniyi ${ }^{3}$, Frederick W. Unverzagt ${ }^{1}$, Hugh C. Hendrie ${ }^{1,2},{ }^{1}$ Indiana University School of Medicine, Indianapolis, IN, USA; ${ }^{2}$ Regenstrief Institute, Indianapolis, IN, USA; ${ }^{3}$ University of Ibadan, Nigeria. Contact e-mail: khall@iupui.edu

Background: Since our 1992 prevalence study there have been advances in medications prescribed in the US for cardiovascular risk factors, while at the same time there has been public health focus on healthful life styles. Cardiovascular risk factors are now thought to confer risk for development of Alzheimer's disease, leading to speculation that rates Alzheimer's disease (AD) may be declining. We compare prevalence rates for dementia and Alzheimer's disease in elderly African Americans in Indianapolis in 2001 and 1992. Methods: This is a 2001 prevalence study of a sample of 1893 African Americans age $\geq 70$ years living independently in the community. The study follows the same two stage design and methods as were used in 1992. In Stage 1 all participants were given the in-home Community Screening Interview for Dementia (CSI-D). CSI-D scores were grouped into good performance, intermediate performance and poor performance for sampling subjects for Stage 2, a full in- home clinical assessment. Stage 2 includes the Consortium to Establish a Registry for Alzheimer's Disease (CERAD) neuropsychological test battery, standardized physical, neurological, and functional assessment using the Clinician Home-based Interview to Assess Function (CHIF), and the informant interview based upon the Cambridge Examination for Mental Disorders in the Elderly. Diagnoses were made in a consensus diagnostic conference using standard criteria. We compare age specific prevalence rates for two non overlapping cohorts assembled at two points in time. Results: The overall age adjusted prevalence rate for dementia for the 2001 cohort was $7.38 \%$ (95\% CI, 4.15-10.60) and for the 1992 cohort was 6.75\% (95\% CI, 5.77-7.74). The overall age adjusted prevalence rate for AD for the 2001 cohort was $6.77 \%$ (95\% CI, 3.65-9.90) and for the 1992 cohort was $5.47 \%$ (95\% CI, 4.51-6.42). The rates for dementia and AD were not significantly different for these two cohorts. Prevalence rates increased with age in both cohorts. Conclusions: Although we have observed increases in prescriptions for reducing cardiovascular risk factors in the nine years since our first prevalence study we do not observe a declining rate in the prevalence of dementia or $\mathrm{AD}$ in these elderly African American cohorts.

\section{4-05-07 DEMENTIA AND DEATH: THE EFFECT OF MORTALITY ON LIFETIME RISKS OF DEMENTIA IN A HIGH RISK COHORT OF OLDER MEXICAN AMERICANS}

Mary N. Haan, University of Michigan, Ann Arbor, MI, USA. Contact e-mail:mnhaan@umich.edu

Background: Both dementia and death increase rapidly with age in older populations and so mortality competes with dementia. Projections of cumulative incidence that do not take death into account (right censoring) do not accurately reflect disease experience in an elderly cohort. Objective: In groups at high risk for early mortality from diabetes or hypertension, competing risk of death can result in an underestimate of the future lifetime risk of dementia after age 60 . This estimates the bias for gender, education and nativity. Methods: The Sacramento Area Latino Study on Aging
(SALSA) is a cohort study of community dwelling older Mexican Americans aged 60-101 at baseline in 1998 of whom 50\% were immigrants. This cohort has been followed annually through early 2008 and characterized for dementia incidence (DEM) and significant cognitive impairment (CIND). Results: Type 2 diabetes baseline prevalence was 33\%, metabolic syndrome prevalence was $51 \%$ and $68 \%$ of the cohort had hypertension $(50 \%$ treated $)$. To date, $25 \%(n=451)$ of the cohort has died; age-adjusted dementia/CIND incidence rates are 12 per 1,000 persons year in men and 14.1 per 1,000 person years in women. Mortality rates are $6.3 / 1,000$ py in men and 6.5/1,000 py in women. The purpose of this analysis is to use a technique published by Beiser (2003) using Framingham data that adjusts cumulative incidence rates for competing risk of death. The effects of this adjustment on rates by gender, country of birth, and education are examined as lifetime risk of 20 years conditional on dementia-free survival to age 60. Adjustment for competing risk of death substantially reduces the projected 20-year lifetime risk for dementia/CIND (Table). The effects of adjustment are greater in men than women, in US-born than Mexican born, and in those with less education ( $<12$ years). These effects increase with aging and length of follow-up (not shown). Conclusions: Comparisons of dementia risk across ethnic groups are affected by differences in competing risk of death. Cross national comparisons are vulnerable to this bias because early mortality varies dramatically between developed and developing countries.

\section{O4-05-08 THE IMPACT OF MIDLIFE SMOKING ON THE DEVELOPMENT OF DEMENTIA AND ALZHEIMER'S DISEASE LATER IN LIFE: A POPULATION-BASED STUDY}

Minna Rusanen ${ }^{1,2}$, Suvi Rovio ${ }^{3}$, Tiia Ngandu ${ }^{3}$, Aulikki Nissinen ${ }^{4}$, Hilkka Soininen ${ }^{1,2}$, Miia Kivipelto ${ }^{3,2},{ }^{1}$ Department of Neurology, Kuopio University Hospital, Kuopio, Finland; ${ }^{2}$ Department of Neuroscience and Neurology, University of Kuopio, Kuopio, Finland; ${ }^{3}$ Aging Research Center, Division of Geriatric Epidemiology, Neurotec, Karolinska Institutet, Stockholm, Sweden; ${ }^{4}$ Department of Epidemiology and Health Promotion, National Public Health Institute, Helsinki, Finland. Contact e-mail: minna.rusanen@uku.fi

Background: Previous findings of the impact of smoking on the risk of dementia and Alzheimer's disease (AD) have been controversial. Nowadays, tobacco is recognized as a predisposing factor to dementia and $\mathrm{AD}$. It has also been suggested that the effect of smoking may differ between APOE $\epsilon 4$ carriers and non-carriers. Our study evaluates the effect of smoking on the risk of dementia and $\mathrm{AD}$ in a large, population based prospective study. Methods: Cardiovascular Risk Factors, Aging and Dementia (CAIDE) study in eastern Finland includes 2000 participants who were randomly selected from four separate population-based samples originally studied in midlife (1972, 1977, 1982 or 1988). After an average follow-up time of 21 years altogether1449 (73\%) individuals aged 65-79 years took part in a re-examination in 1998. 61 persons were diagnosed with dementia and 48 had AD. In addition, dementia diagnoses of the non-participants were derived from the patient records of local hospitals and health centres. Consequently, the total number of persons with dementia and AD increased to 117 and 76, respectively. Smoking was assessed in midlife with a detailed questionnaire, and the subjects were classified as current, former or non-smokers. Also pack-years (the average number of cigarettes daily smoked divided by 20 and multiplied by the number of years of smoking) were determined. Results: Smoking in midlife increased the risk of dementia (OR 4.93, 95\% CI 1.51-16.11) and $\mathrm{AD}$ (OR 6.56, 95\% CI 1.80-23.94) among APOE $\epsilon 4$ carriers after adjustment for sociodemographic risk factors, vascular risk factors and events and lung diseases. The risk was more pronounced among those APOE $\epsilon 4$ carriers who smoked and also frequently drank alcohol (for dementia OR 17.24, 95\% CI 1.95-152.37 and for AD OR 11.05, 95\% CI 1.08-113.30). There was no association found between smoking and dementia or AD among APOE $\epsilon 4$ non-carriers. Pack-years were not related to dementia. Conclusions: In this study smoking was found to increase the risk of dementia and AD only among APOE $\epsilon 4$ carriers. These 